\title{
Serum Cytokine Levels for Predicting Immune- Related Adverse Events and the Clinical Response in Lung Cancer Treated with Immunotherapy
}

Ni Zhao

The First Affiliated Hospital of Xi'an Jiaotong University

Ye Yi

The First Affiliated Hospital of Xi'an Jiaotong University

Wen Cao

The First Affiliated Hospital of Xi'an Jiaotong University

Nan Mei

The First Affiliated Hospital of Xi'an Jiaotong University

Xiao Fu

The First Affiliated Hospital of Xi'an Jiaotong University

Chunli Li ( $\nabla$ chunli5158@163.com )

The First Affiliated Hospital of Xi'an Jiaotong University

\section{Research Article}

Keywords: biomarkers, cytokines, immunotherapy, cancer

Posted Date: January 12th, 2022

DOI: https://doi.org/10.21203/rs.3.rs-1212304/v1

License: (c) (1) This work is licensed under a Creative Commons Attribution 4.0 International License.

Read Full License 


\section{Abstract}

Analyses of the composition of peripheral cytokines hold promise for providing a basis for determining the prognosis of lung cancer treated with immunotherapy. In this study, we assessed correlations between interleukins in peripheral blood and the disease prognosis in patients with lung cancer. We retrospectively collected eligible adult patients with histologically confirmed lung cancer. Patients with immune-related adverse events (AE) from immunotherapy had higher pretreatment levels of IL-2 $(p=0.002), I L-17(p=0.01)$, and IFN- $a(p=0.02)$ than patients with nonimmune-related adverse events (NAE). Univariate analysis showed that changes in IL-2 ( $p=0.04), I L-5(p=0.007), I F N-a(p=0.003), I F N-\gamma$ $(p=0.012)$ and TNF- $a(p=0.049)$ levels were significantly increased in patients with AE compared with those with NAE before the second cycle of therapy. Patients with a clinical benefit had higher levels of IL17 before the third cycle than patients without a clinical benefit. No significant cytokine differences were observed between patients with and without a clinical benefit undergoing $\mathrm{ICl}$ pretreatment or in the first two cycles of therapy. Plasma cytokines are related to immune-related adverse events and clinical responses, which are potential predictive markers for anti-PD-1/PD-L1 therapy in lung cancer patients and may play an important role in selecting patients who would benefit from PD-1/PD-L1 inhibitors.

\section{Introduction}

Lung cancer is the leading cause of cancer-related deaths worldwide. ${ }^{1}$ Treatment with immune checkpoint inhibitors (ICls) has led to a paradigm shift in the treatment of solid tumors, including lung cancer. ${ }^{2-4}$ Although recent clinical studies have demonstrated that PD-L1 expression on tumor cells is associated with clinical benefits in the treatment of lung cancer, ${ }^{3,5}$ anti PD-1/PD-L1 immunotherapy is also effective in some patients whose PD-L1 levels are low in their tumor tissue. 2,4 Moreover, because of the difficulty associated with obtaining tumor tissues, the identification of prognostic biomarkers in circulating blood for patient selection in pragmatic clinical settings would be of considerable value for optimizing and personalizing anti-PD-1/PD-L1 immunotherapy. Some reports have also suggested that the tumor mutational burden (TMB), the neoantigen burden and the presence of tissue infiltrating lymphocytes are predictive biomarkers in $\mathrm{ICl}$ treatment. ${ }^{6,7}$ Moreover, an increasing number of preclinical and clinical studies have suggested that infiltrating immune cells within a tumor or the tumor cells themselves produce cytokines and chemokines, leading to modulation of the tumor microenvironment and promoting angiogenesis, growth, invasion and metastasis. ${ }^{8}$ In this study, we explored the biomarkers associated with clinical benefits such as tumor response and onset of irAEs(Immune-related Adverse Events). The aim of our study was to investigate whether a defined cytokine panel (IL-1 $\beta$, IL-2, IL-4, IL-5, IL6 , IL-8, IL-10, IL-12, IL-20, IFN-a, IFN- - , TNF-a) can play a prognostic or predictive role in lung cancer patients treated with immune checkpoint inhibitors to assess any potential correlations between their serum levels and clinical safety and the treatment response.

\section{Results}




\section{Patient characteristics:}

We prospectively analyzed 229 patients treated at the First Affiliated Hospital of Xi'an Jiaotong University from November 2020 to September 2021 (Table 1). Of these, 192 (84\%) patients were male and 16 (27\%) were female, with a median age of 64 years (range 34-81). The most frequent histological types were adenocarcinoma (39\%), squamous carcinoma (34\%), and small cell lung cancer (21\%). Among those eligible for evaluation, 117 patients (51\%) had lymph node metastasis, and 82 patients had bone metastasis. The median line of therapy received was 1 , and the most common ICls used were pembrolizumab (23\%), tislelizumab (24\%) and camrelizumab (19\%). A total of 167 patients $(73 \%)$ completed more than 3 cycles of therapy. 
Table 1

Summary of patient baseline characteristics

\begin{tabular}{|ll|}
\hline & Overall population, N =229(\%) \\
\hline Age & $64(34-81)$ \\
\hline Sex & \\
\hline Male & $192(84 \%)$ \\
\hline Female & $37(16 \%)$ \\
\hline Smoke Status & \\
\hline Current or former smoker & $144(63 \%)$ \\
\hline Never smoker & $85(37 \%)$ \\
\hline Hypertension & \\
\hline Yes & $57(25 \%)$ \\
\hline No & $172(75 \%)$ \\
\hline Diabetes & \\
\hline Yes & $19(8 \%)$ \\
\hline No & $210(92 \%)$ \\
\hline Histology & \\
\hline Adenocarcinoma & $89(39 \%)$ \\
\hline Squamous carcinoma & $79(34 \%)$ \\
\hline Small cell lung cancer & $49(21 \%)$ \\
\hline Other & $12(6 \%)$ \\
\hline Metastatic number & \\
\hline$<2$ & $55(24 \%)$ \\
\hline$\geq 2$ & $62(76 \%)$ \\
\hline PD-L1 expression & \\
\hline Positive & \\
\hline Negative & \\
\hline Unknown & \\
\hline Metastases Organ* & \\
\hline
\end{tabular}




\begin{tabular}{|ll|}
\hline & Overall population, N =229(\%) \\
\hline Liver metastasis & $44(19 \%)$ \\
\hline Bone metastasis & $82(36 \%)$ \\
\hline Brain metastasis & $43(19 \%)$ \\
\hline Lung metastasis & $63(28 \%)$ \\
\hline Lymph node metastasis & $117(51 \%)$ \\
\hline Other organ metastasis & $57(25 \%)$ \\
\hline ICl category & \\
\hline PD-1 ICl & $188(82 \%)$ \\
\hline PD-L1 ICI & $41(18 \%)$ \\
\hline ICl treatment received & \\
\hline Pembrolizumab & $52(23 \%)$ \\
\hline Atezolizumab & $16(7 \%)$ \\
\hline Toripalimab & $12(5 \%)$ \\
\hline Camrelizumab & $43(19 \%)$ \\
\hline Tislelizumab & $54(24 \%)$ \\
\hline Durvalumab & $25(11 \%)$ \\
\hline Sintilimab & $26(11 \%)$ \\
\hline Line of therapy & \\
\hline First line & $160(70 \%)$ \\
\hline Second line & $58(25 \%)$ \\
\hline Third line & $8(3 \%)$ \\
\hline Further lines & \\
\hline DOS & \\
\hline S3 & \\
\hline$>3$ & \\
\hline
\end{tabular}


First, we evaluated the potential of serum proteins as predictive biomarkers of irAE onset. The most frequently reported immune-related adverse events were checkpoint inhibitor-related pneumonitis (31, $13.5 \%)$, myocarditis $(12,5 \%)$, and hypothyroidism $(11,4.8 \%)$, and 2 of these patients died because of acute adverse events. Cytokine data were available at baseline and at 1 month for 110 and 65 patients, respectively.

Patients with AEs from immunotherapy had higher pretreatment levels of IL-2 ( $p=0.002), I L-17(p=0.01)$, and IFN-a $(p=0.02)$ than patients with NAEs (Table 2a and Figure 2).

Table 2

a. Cytokine levels with clinical safety at baseline and cycle 1 therapy.

\begin{tabular}{|lllll|}
\hline Pretreatment & \multicolumn{5}{l|}{} \\
\hline & Univariate & Multivariate & \\
\cline { 2 - 5 } & $p$ & Odds Ratio & OR $(95 \% \mathrm{Cl})$ & $p$ \\
\hline IL-2 & 0.004 & 1.592 & $1.165-2.176$ & 0.002 \\
\hline IL-17 & 0.397 & 1.224 & $1.048-1.430$ & 0.011 \\
\hline TNF-a & 0.025 & 1.127 & $1.026-1.250$ & 0.023 \\
\hline On-treatment & & & & \\
\hline IL-2 & 0.118 & 1.260 & $1.010-1.572$ & 0.040 \\
\hline IL-5 & 0.019 & 1.237 & $1.059-1.445$ & 0.007 \\
\hline IFN-a & 0.004 & 1.451 & $1.133-1.858$ & 0.003 \\
\hline IFN-y & 0.015 & 1.064 & $1.014-1.116$ & 0.012 \\
\hline TNF-a & 0.067 & 1.128 & $1.007-1.271$ & 0.049 \\
\hline
\end{tabular}

Table 2

b. Cytokine levels with clinical safety before cycle 3 therapy.

\begin{tabular}{|lllll|}
\hline On-treatment & \multicolumn{5}{l|}{} \\
& Univariate & Multivariate & & \\
\cline { 2 - 5 } & $p$ & Odds Ratio & OR $(95 \% \mathrm{Cl})$ & $p$ \\
$\mathrm{IL}-10$ & 0.041 & 0.574 & $0.338-0.976$ & 0.143 \\
$\mathrm{IL}-17$ & 0.028 & 0.815 & $0.673-0.986$ & 0.035 \\
\hline
\end{tabular}

Table 2. Associations of (a) pretreatment and (b) on-treatment (before second cycle of therapy) cytokine levels with clinical response from systemic therapy. The $\chi 2$ test was used to determine statistical 
significance in comparison of high versus low cytokine values between $A E$ and NAE patients. $A E$, adverse events; NAE, nonadverse events; IFN- - , interferon- $\gamma$; IL, interleukin; TNF, tumor necrosis factor.

Additionally, we assessed cytokine concentration changes before the second cycle of therapy. Univariate analysis showed that changes in IL-2 ( $p=0.04)$, IL-5 $(p=0.007)$, IFN- $a(p=0.003)$, IFN-y $(p=0.012)$ and TNF-a $(p=0.049)$ levels were significantly increased in patients with irAEs compared with those without irAEs before the second cycle of therapy (Table 2a and Figure 3).

\section{Clinical response/treatment efficacy:}

Next, we evaluated the potential of serum proteins as predictive biomarkers of the ciliary response. At the time of analysis, the median PFS for all patients was 3.3 months $(95 \% \mathrm{Cl}, 2.9-4.0)$, and OS could not be analyzed. Patients with CB had higher levels of IL-17 before the third cycle than patients with NCB (Table $2 \mathrm{~b}$ and Figure 4). No significant cytokine differences were noted among $\mathrm{CB}$ and NCB patients undergoing $\mathrm{ICl}$ pretreatment or in the first two cycles of therapy.

\section{Discussion}

This is the first retrospective study involving analyses of baseline and on-treatment cytokine concentrations during ICl therapy. We found that pretreatment levels of IL-2, IL-17, and TNF-a as well as on-treatment levels of IL-2, IL-17, IFN- $a$, IFN- $y$, and TNF-a were associated with immune-related adverse events. At the same time, on-treatment levels of IL-17 were related to the clinical response.

Growing evidence indicates that immunotoxicity profiles can be tied to specific cytokines that can amplify both pro- and anti-inflammatory immunity. ${ }^{10}$ Among Th2 cytokines, IL-2 is a key cytokine involved in promoting the proliferation of natural killer (NK) cells and T lymphocytes. ${ }^{11}$ Constantini et al. ${ }^{12}$ showed that a low serum IL-2 concentration measured at nivolumab initiation was associated with grade 3-4 toxicities in patients with advanced NSCLC; however, no association with progression-free survival (PFS) or overall survival (OS) was observed.

IL-5 is mainly produced by T helper-2 (Th2) lymphocytes and Group 2 innate lymphoid cells (ILC2s). It can increase antibody secretion by promoting the differentiation and growth of $B$ cells and enhance the humoral immune response mediated by Th2 cells. Immunity to tumors is mainly governed by Th1mediated cellular immunity. A Th1-Th2 drift will lead to immunosuppression and cancer development. ${ }^{13}$

High concentrations of baseline serum IL-17 were identified in ipilimumab (anti-CTLA-4 Ab)-treated metastatic melanoma patients developing severe grade 3 gastrointestinal irAEs and may thus serve as a putative biomarker for defining both at-risk patients and the severity of ipilimumab-induced colitis. ${ }^{14}$

With close collaborations between academia and industry, recombinant IFNa2 became the first human immunotherapeutic approved by the US Food and Drug Administration (FDA) for cancer and, other than insulin, the first FDA-approved pharmaceutical product produced by recombinant DNA technology. ${ }^{15}$ IFNa 
has multiple antitumor properties, including direct tumor cell killing and stimulation of host immune cells, including dendritic cells and CD8+ T cells. ${ }^{16-18}$ However, no association has been found between the level of IFN-a and immune-related adverse events. According to our results, we can explain why overactivated immune cells can also damage other normal cells, which may lead to immune-related adverse events.

IFN- $y$ has various roles in immune reactions against tumors, including stimulation of tumor-infiltrating lymphocyte (TIL) proliferation and differentiation and secretion of IFN- $\gamma$ following activation of $T$ lymphocytes by tumor antigens. ${ }^{19}$ In contrast, IFN- $\gamma$ may also promote the production of immunosuppressive molecules, which can have direct negative feedback on effector T cell function. ${ }^{20}$ During the elimination phase of the immune response against tumor cells, recruited tumor-infiltrating macrophages and NK cells produce various cytokines, including IFN- - , to kill tumor cells. ${ }^{21}$ Therefore, an elevated level of IFN- $\gamma$ may suggest increased cytotoxic activity against lung cancer tumor cells. However, this mechanism of action can also give rise to autoimmune-like side effects known as irAEs. In a study by Constantini et al. ${ }^{12}$ IFN-y levels at nivolumab initiation and two months later did not show correlations with the objective response rate, clinical benefit, or survival, which is consistent with our study.

In the tumor microenvironment, TNF-a acts as an inflammatory mediator involved in tumorigenesis. ${ }^{22,23}$ Soluble TNF-a is also involved in the activation of neutrophils, macrophages, and lymphocytes at damaged and infected sites ${ }^{24}$. In rheumatoid arthritis, the inflammatory response is associated with increased secretion of tumor necrosis factor (TNF)- $a$ and interleukin (IL)-6, whereas psoriasis involves the production of TNF-a, IL-17, and IL-23, in affected tissues. ${ }^{25}$

Perez-Ruiz et al. ${ }^{26}$ found that enriched TNF gene expression was enriched in colonic mucosal tissue biopsies from CPI-treated cancer patients who developed colonic irAEs compared to healthy controls, which was further confirmed in another study by single-cell RNA sequencing, where melanoma patients who developed CPI-induced colitis displayed an increased abundance of myeloid cells in the colon with an enriched TNF-a gene signature compared to CPI-treated melanoma patients without colitis and to healthy controls. ${ }^{27}$

\section{Conclusion}

Cytokine serum levels may provide prognostic information and constitute predictive markers of immunotherapy benefits in patients with lung cancer. Further studies of the predictive effects of these markers in larger populations are warranted.

\section{Method}

\section{Patient selection and sample acquisition}


This was a single-arm retrospective clinical study. Eligible patients were adults with histologically confirmed lung cancer. Patients with a previous history of systemic immunosuppressive therapy or active autoimmune disease were excluded (Figure 1). Agent choice was based on PD-L1 status and patients' previous treatment history (first- or second-line setting).

\section{Grading of toxicity}

Toxic effects were graded with the use of the National Cancer Institute Common Terminology Criteria for Adverse Events, version 4.0. Scheduled computed tomography or magnetic resonance imaging was performed every 9-12 weeks. Although immune-related response criteria were evaluated, the primary radiographic assessment was carried out using the Response Evaluation Criteria in Solid Tumors (RECIST), version 1.1.

\section{Cytokine testing in blood by flow cytometry}

Peripheral blood samples were included by immune response at the following time points: before anti-PD1 inhibition treatment (every 3 weeks/ 1 cycle, a total of 4 cycles).

The 3-month (4 cycles) follow-up period was determined on the basis of the essential time required for the immune system to convert from innate to adaptive responses. ${ }^{9}$

Blood samples were collected from all patients before starting anti-PD- 1 inhibitors (every 3 weeks/ 1 cycle, a total of 4 cycles). Serum samples were collected and processed using the same standardized protocol. Briefly, blood samples were left to coagulate at room temperature for $30 \mathrm{~min}$ and centrifuged at $1000 \mathrm{~g}$ for $10 \mathrm{~min}$ at room temperature, and the supernatants were collected, divided into aliquots, and stored at $-80^{\circ} \mathrm{C}$ until assay. The time interval between processing and freezing was no more than $2 \mathrm{~h}$ for each sample. None of the samples were thawed more than twice before analysis.

The serum levels of the following cytokines were measured: IL-1 $\beta$, IL-2, IL-4, IL-5, IL-6, IL-8, IL-10, IL-12, IL17, IFN-a, IFN- $y$, and TNF-a. The principle of the direct sandwich method is consistent with the classical sandwich immunoassay. Specifically, $45 \mu \mathrm{l}$ of serum sample and $45 \mu \mathrm{l}$ beads of the panel kit were mixed and then incubated for $1 \mathrm{~h}$ at room temperature. After incubation, $0.5 \mathrm{ml}$ of wash buffer was added, and the samples were centrifuged for $5 \mathrm{~min}$. Samples were incubated first with biotin-conjugated monoclonal antibody (30 $\mathrm{min}$ ) and then subsequently incubated with streptavidin-conjugated monoclonal antibody (20 min). Finally, wash reading buffer was added to all samples. The technical principle is that the cytokine antibody conjugated by fluorescein microspheres is combined with the biotin-labeled cytokine paired antibody and the cytokines in the sample or calibration to form a "sandwich" complex, which then reacts with the addition of phyoglobinin-labeled streptavidin (SA-PE). The fluorescence intensity was directly proportional to the cytokine content in the sample within the detection range.

\section{Abbreviations}


ICls Immune checkpoint inhibitors

IFN-ץ Interferon gamma

IL Interleukin

IL-1RA Interleukin 1 receptor antagonist

IP10 Interferon gamma-induced protein 10

irAEs Immune-related adverse events

PD-1 Programmed cell death protein 1

PD-L1 Programmed death-ligand 1

TNF-a Tumor necrosis factor alpha

TNF-y Tumor necrosis factor gamma

CTCAE Common Terminology Criteria for Adverse Events

RECIST Response Evaluation Criteria in Solid Tumors

ICls Immune checkpoint inhibitors

TMB Tumor mutational burden

CB Clinical benefit

NCB No clinical benefit

PR partial response

SD stable disease

PD progressive disease

PFS Progression-free survival

AE Adverse events

NAE Non-adverse events

NK Natural killer

NSCLC Non-small cell lung cancer 
CTLA-4 Cytotoxic T lymphocyte-associated antigen-4

FDA Food and Drug Administration

TILs Tumor-infiltrating lymphocyte

CPI Checkpoint inhibitor

\section{Declarations}

Contributors: Study concept and design: N.Z. and C.L. Acquisition, analysis, or interpretation of data: Y.Y., W.C., X.F. and N.M. Drafting of the manuscript: N.Z. Critical revision of the manuscript for important intellectual content: Y.Y., W.C. and N.M. Statistical analysis: N. Z.

Funding: This study was supported by National Natural Science Foundation of China (Grant No. 82002437).

Conflict of interest: The authors declare that they have no any conflicts of interest.

Provenance and peer review: Not commissioned; externally peer reviewed.

Data availability statement: Data are unavailable.

\section{References}

1. Sung, H. et al. Global Cancer Statistics 2020: GLOBOCAN Estimates of Incidence and Mortality Worldwide for 36 Cancers in 185 Countries. CA Cancer J Clin 71, 209-249, doi:10.3322/caac.21660 (2021).

2. Borghaei, H. et al. Nivolumab versus Docetaxel in Advanced Nonsquamous Non-Small-Cell Lung Cancer. N Engl J Med 373, 1627-1639, doi:10.1056/NEJMoa1507643 (2015).

3. Reck, M. et al. Pembrolizumab versus Chemotherapy for PD-L1-Positive Non-Small-Cell Lung Cancer. N Engl J Med 375, 1823-1833, doi:10.1056/NEJMoa1606774 (2016).

4. Herbst, R. S. et al. Pembrolizumab versus docetaxel for previously treated, PD-L1-positive, advanced non-small-cell lung cancer (KEYNOTE-010): a randomised controlled trial. Lancet 387, 1540-1550, doi:10.1016/s0140-6736(15)01281-7 (2016).

5. Brahmer, J. et al. Nivolumab versus Docetaxel in Advanced Squamous-Cell Non-Small-Cell Lung Cancer. N Engl J Med 373, 123-135, doi:10.1056/NEJMoa1504627 (2015).

6. Yarchoan, M., Hopkins, A. \& Jaffee, E. M. Tumor Mutational Burden and Response Rate to PD-1 Inhibition. N Engl J Med 377, 2500-2501, doi:10.1056/NEJMc1713444 (2017).

7. Tumeh, P. C. et al. PD-1 blockade induces responses by inhibiting adaptive immune resistance. Nature 515, 568-571, doi:10.1038/nature13954 (2014). 
8. Grivennikov, S. I., Greten, F. R. \& Karin, M. Immunity, inflammation, and cancer. Cell 140, 883-899, doi:10.1016/j.cell.2010.01.025 (2010).

9. Ziauddin, J. \& Schneider, D. S. Where does innate immunity stop and adaptive immunity begin? Cell Host Microbe 12, 394-395, doi:10.1016/j.chom.2012.10.004 (2012).

10. Kang, J. H., Bluestone, J. A. \& Young, A. Predicting and Preventing Immune Checkpoint Inhibitor Toxicity: Targeting Cytokines. Trends Immunol 42, 293-311, doi:10.1016/j.it.2021.02.006 (2021).

11. Berraondo, P. et al. Cytokines in clinical cancer immunotherapy. Br J Cancer 120, 6-15, doi:10.1038/s41416-018-0328-y (2019).

12. Costantini, A. et al. Predictive role of plasmatic biomarkers in advanced non-small cell lung cancer treated by nivolumab. Oncoimmunology 7, e1452581, doi:10.1080/2162402x.2018.1452581 (2018).

13. Ikutani, M. et al. Identification of innate IL-5-producing cells and their role in lung eosinophil regulation and antitumor immunity. J Immunol 188, 703-713, doi:10.4049/jimmunol.1101270 (2012).

14. Tarhini, A. A. et al. Baseline circulating IL-17 predicts toxicity while TGF- $\beta 1$ and IL-10 are prognostic of relapse in ipilimumab neoadjuvant therapy of melanoma. J Immunother Cancer 3, 39, doi:10.1186/s40425-015-0081-1 (2015).

15. Borden, E. C. Interferons $a$ and $\beta$ in cancer: therapeutic opportunities from new insights. Nat Rev Drug Discov 18, 219-234, doi:10.1038/s41573-018-0011-2 (2019).

16. Papageorgiou, A., Dinney, C. P. \& McConkey, D. J. Interferon-alpha induces TRAIL expression and cell death via an IRF-1-dependent mechanism in human bladder cancer cells. Cancer Biol Ther 6, 872879, doi:10.4161/cbt.6.6.4088 (2007).

17. Schiavoni, G., Mattei, F. \& Gabriele, L. Type I Interferons as Stimulators of DC-Mediated CrossPriming: Impact on Anti-Tumor Response. Front Immuno/ 4, 483, doi:10.3389/fimmu.2013.00483 (2013).

18. Jennings, R. N., Grayson, J. M. \& Barton, E. S. Type I interferon signaling enhances CD8+ T cell effector function and differentiation during murine gammaherpesvirus 68 infection. $J$ Viro/ 88, 14040-14049, doi:10.1128/jvi.02360-14 (2014).

19. Karachaliou, N. et al. Interferon gamma, an important marker of response to immune checkpoint blockade in non-small cell lung cancer and melanoma patients. Ther Adv Med Oncol 10, 1758834017749748, doi:10.1177/1758834017749748 (2018).

20. Gajewski, T. F., Schreiber, H. \& Fu, Y. X. Innate and adaptive immune cells in the tumor microenvironment. Nat Immunol 14, 1014-1022, doi:10.1038/ni.2703 (2013).

21. Ma, W., Gilligan, B. M., Yuan, J. \& Li, T. Current status and perspectives in translational biomarker research for PD-1/PD-L1 immune checkpoint blockade therapy. J Hematol Oncol 9, 47, doi:10.1186/s13045-016-0277-y (2016).

22. Landskron, G., De la Fuente, M., Thuwajit, P., Thuwajit, C. \& Hermoso, M. A. Chronic inflammation and cytokines in the tumor microenvironment. J Immunol Res 2014, 149185, doi:10.1155/2014/149185 (2014). 
23. Szlosarek, P., Charles, K. A. \& Balkwill, F. R. Tumour necrosis factor-alpha as a tumour promoter. Eur J Cancer 42, 745-750, doi:10.1016/j.ejca.2006.01.012 (2006).

24. Sethi, G., Sung, B. \& Aggarwal, B. B. TNF: a master switch for inflammation to cancer. Front Biosci 13, 5094-5107, doi:10.2741/3066 (2008).

25. Mclnnes, I. B., Buckley, C. D. \& Isaacs, J. D. Cytokines in rheumatoid arthritis - shaping the immunological landscape. Nat Rev Rheumatol 12, 63-68, doi:10.1038/nrrheum.2015.171 (2016).

26. Perez-Ruiz, E. et al. Prophylactic TNF blockade uncouples efficacy and toxicity in dual CTLA-4 and PD-1 immunotherapy. Nature 569, 428-432, doi:10.1038/s41586-019-1162-y (2019).

27. Luoma, A. M. et al. Molecular Pathways of Colon Inflammation Induced by Cancer Immunotherapy. Cell 182, 655-

671.e622

, doi:10.1016/j.cell.2020.06.001 (2020).

\section{Figures}




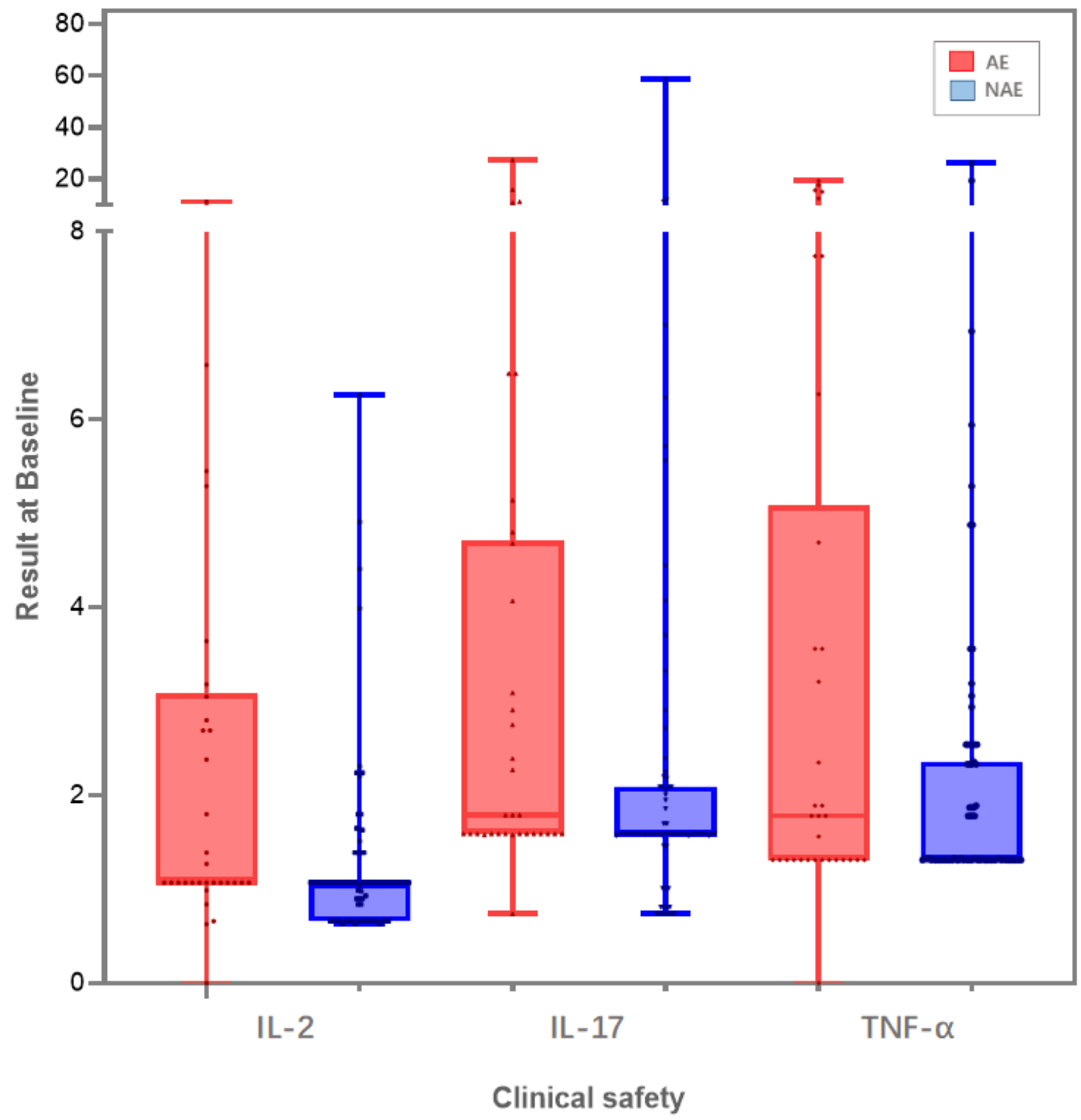

Figure 1

Selection process for patients 


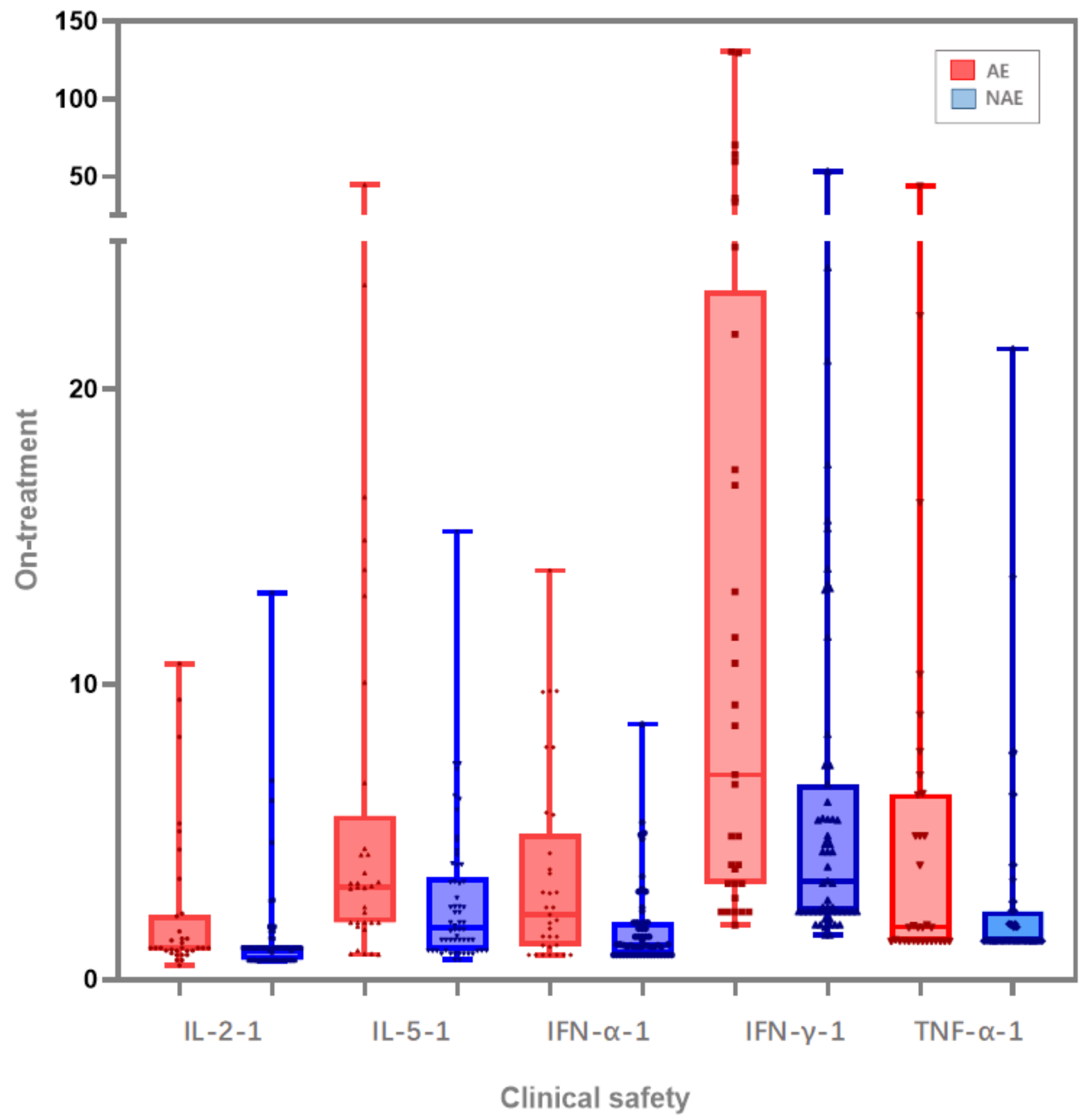

Figure 2

Associations between pretreatment interleukin-2 (IL-2), IL-17 and interferon- $\gamma$ (IFN- $\gamma$ ). Box contains values q1, median and q2. Whiskers expand out to 10th and 90th percentiles. AE, immune-related adverse events; NAE, nonimmune-related adverse events 


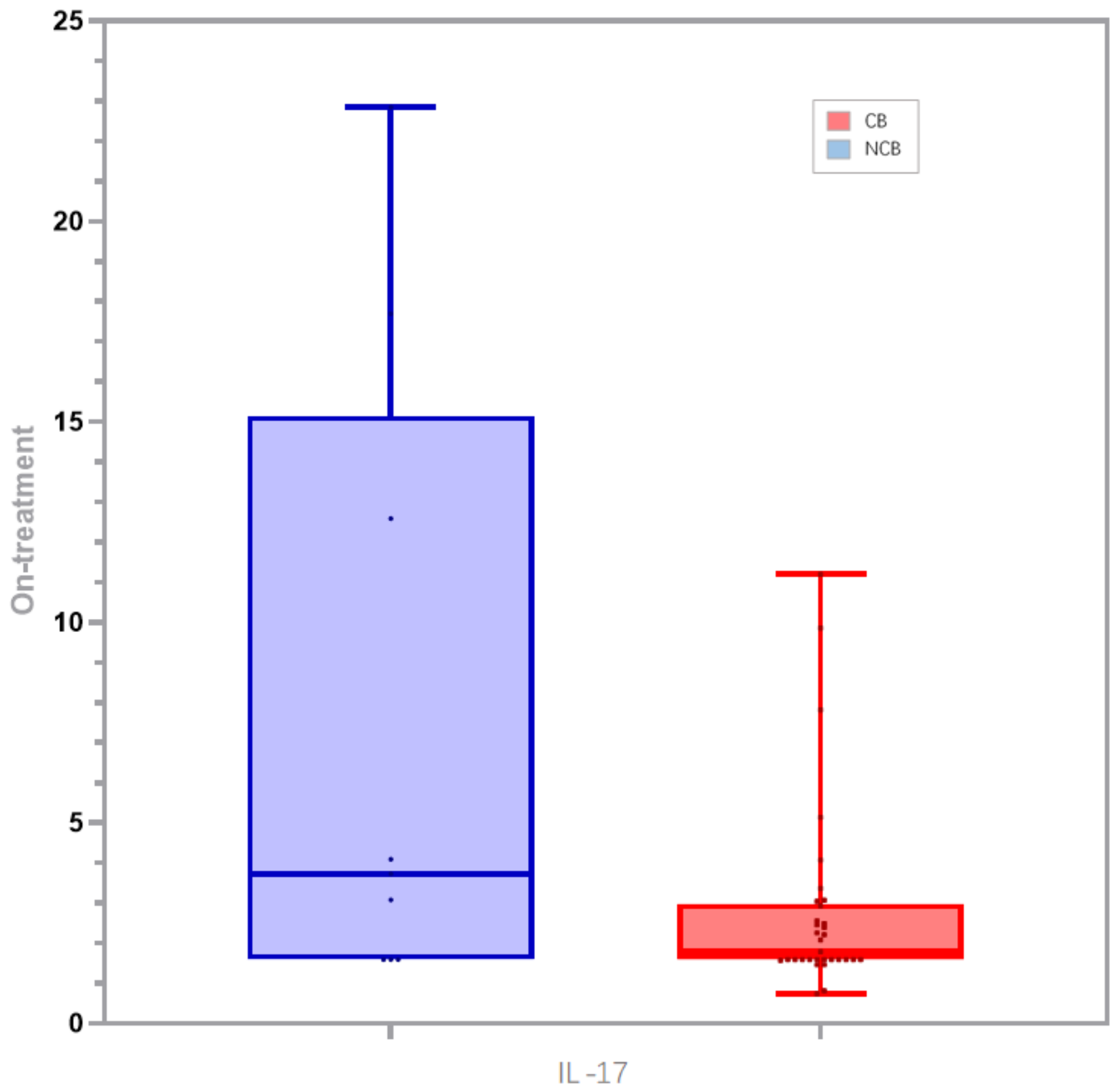

Clinical response

Figure 3

Associations between on-treatment (before second cycle of therapy) IL-2, IL-5, interferon-a (IFN- $a$ ), IFN- $\gamma$ and tumor necrosis factor (TNF-a). Box contains values q1, median and q2. AE, adverse events; NAE, nonadverse events. 
Lung cancer patients treated with immune checkpoint inhibitors from November 2020 to September 2021 at the First Affiliated Hospital of Xi 'an Jiaotong University

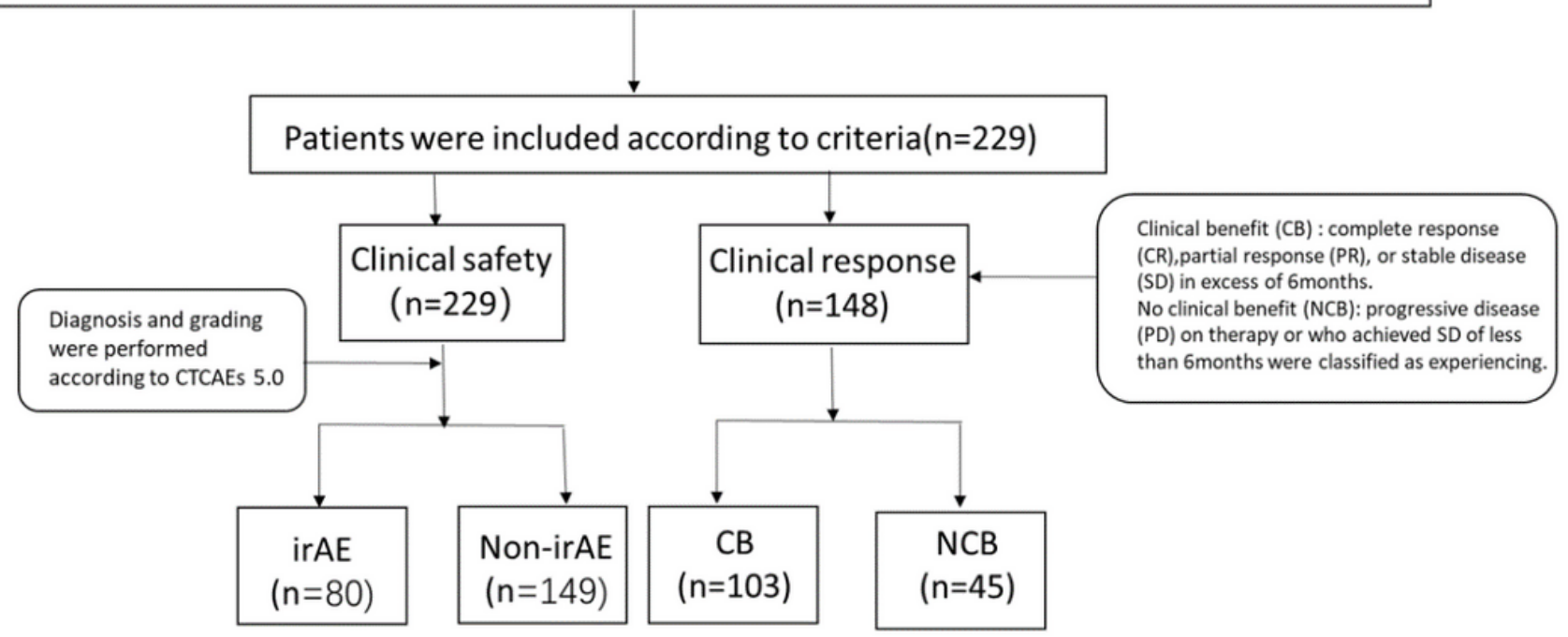

Figure 4

Associations between on-treatment (before the third cycle) IL-17 changes. Box contains values q1, median and q2. Whiskers expand out to 10th and 90th percentiles. 